\title{
Small Epidermal UHF RFID Loop Antenna for Passive Oral Cavity Control Applications and Patient Health Monitoring
}

\author{
P.S. Taylor and J.C. Batchelor \\ Department of Engineering and Digital Arts, University of Kent, Canterbury, Kent, UK. p.taylor-250@kent.ac.uk
}

\begin{abstract}
This paper introduces small loop type antennas and their potential for use in UHF RFID far-field applications. They are typically known as 'magnetic loops' and an established antenna for HF applications. Here we evaluate their potential for use, both by simulation and measurement in the challenging environment of the oral cavity for patient health monitoring and assistive technologies.
\end{abstract}

Keywords-RFID; antenna; loop; magnetic; healthcare; patient; epidermal; stl; assistive technologies;

\section{INTRODUCTION}

Small transmitting loops (STLs), also referred to as 'magnetic loops' [1] are compact efficient antennas. Their main applications have been in HF communications, where wavelengths are large, therefore providing an effective radiator that occupies a relatively small footprint when compared to more conventional antennas such as dipoles and full-size loops. The use of STLs at UHF frequencies and above - where antennas might already seem compact due to the short wavelengths may have previously been an unnecessary requirement. With advances in passive UHF RFID sensors and a need for ever smaller electronics, particularly for body-worn applications the antenna dominates the sensor, potentially limiting optimal placement dues to its physical size. This paper discusses basic STL operation and demonstrates by simulation and measurement a UHF RFID based STL placed upon the hard palate of the mouth. This oral placement would find applications for tongue controlled assistive technologies or patient health monitoring applications [2-3]. It is noted that, although these are compact antenna types they are for operation in the far-field, providing greater read ranges than the few centimetres a near-field system typically offers.

\section{THEORY OF OPERATION}

\section{A. Small Transmitting Loop}

A single turn circular STL is considered small when its conductor length is $<\lambda / 10$, this results in a near uniform in-phase current distribution around the loops perimeter. The loop therefore behaves as a lumped inductance which can be conveniently resonated at the frequency of interest with a capacitor, forming a high-Q parallel tuned circuit and resulting in a narrow bandwidth. The radiation resistance of an STL is very small, and consequently the loops ohmic and skin effect losses must be kept low to maximise its radiation efficiency. Shown in Fig. 1 is an STL and its equivalent circuit. A vertically mounted STL exhibits a figure of eight radiation pattern with maxima in the plane of the loop and sharp nulls at right angles as shown in Fig. 2.

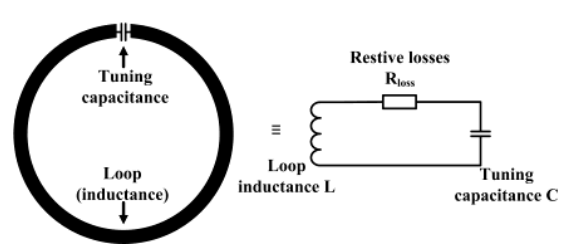

Fig. 1. Small trnasmitting loop and its equivalent circuit

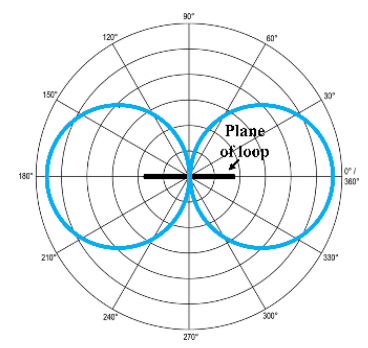

Fig. 2. Radiation pattern for an STL 


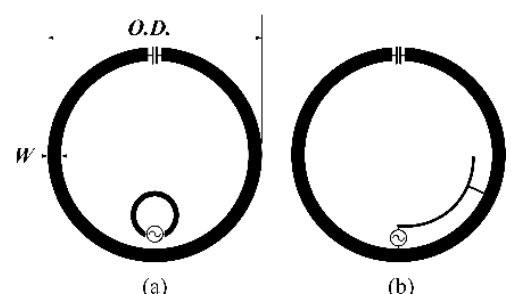

Fig. 3. STL feed arrangements, loop couple shown in (a) and the gamma match in (b)

\section{B. Feeding the Loop}

Several methods can be used to couple a source to an STL, the common ones are the loop couple and gamma match as shown in Fig. 3. The loop couple method consists of a smaller loop, typically one fifth the diameter of the main loop, placed at the loops neutral point opposite the tuning capacitor. This provides a transformer action and a good match over the operational bandwidth of the loop. The second is the gamma match, where a section of conductor is placed parallel to the main loop, fed at its neutral point and a shorting bar is adjusted to achieve an optimum match to the source, typically $50 \Omega$.

\section{SimUlation AND MEASUREMENTS}

Using CST Microwave Studio ${ }^{\mathrm{TM}}$ (CST MWS $^{\mathrm{TM}}$ ) an experimental STL was designed for the European RFID band of $866 \mathrm{MHz}$.

\section{A. Loop Design and Simulation}

The design uses the Alien Higgs 3 RFID device which has an input impedance of 27 - j195 $\Omega$ and an input sensitivity of $-15 \mathrm{dBm}$ at this frequency. The gamma match was the chosen feed method as this proves more versatile in cancelling out the capacitive reactance of the RFID device, whilst still providing a good match to its real part. With reference to Fig. 3 the experimental design dimensions were O.D. $=10 \mathrm{~mm}(\sim \lambda / 10), W=1.5 \mathrm{~mm}$, gamma width of $1 \mathrm{~mm}$ with a $1.5 \mathrm{~mm}$ spacing from the main loop conductor. The design uses copper foil of thickness $0.066 \mathrm{~mm}$ as its conductor. A mouth model was imported into CST MWS ${ }^{\mathrm{TM}}$ consisting of all the main components of the human mouth and their properties. Using a $0.5 \mathrm{~mm}$ thick polymide substrate the design was placed on the hard palate of the mouth as shown in Fig. 4(a). Initial simulations yielded a loop inductance of $15 \mathrm{nH}$, resulting in a capacitance of $2.2 \mathrm{pF}$ to resonate it at $866 \mathrm{MHz}$. This gave a return loss of $-11 \mathrm{~dB}$ and a radiation efficiency of $-16 \mathrm{~dB}$. The optimal position of the shorting bar for the gamma match was found to be $\sim 36^{\circ}$ counter clockwise from the feed point.

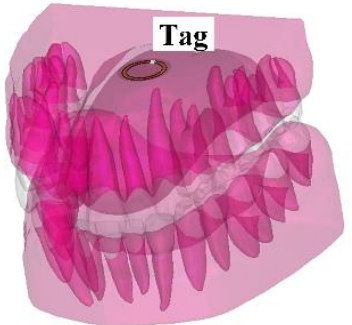

(a)

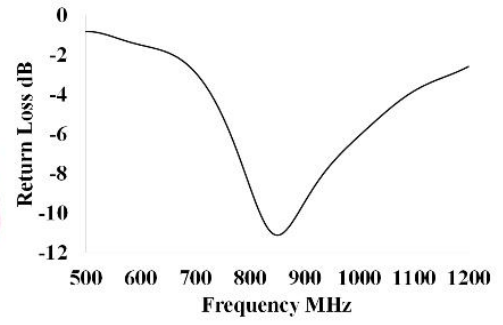

(b)

Fig. 4. CST MWS ${ }^{\mathrm{TM}}$ mouth model with palate attached STL shown in (a) and its simulated Return Loss $\mathrm{S}_{11}$ in (b)

\section{B. Loop Construction and Practical Measurements}

An STL was constructed to the dimensions previously given. A surface mount trimmer capacitor of 1.5-3pF was used to enable tuning of the loop. In practice this would be a fixed value or formed from the loop conductor ends as an interdigital capacitor once the value is determined. The loop was given a polythene covering to protect it from moisture, whilst still giving access to the gamma match and trimmer capacitor for fine tuning. A product called Fixodent (denture adhesive) was used to attach the loop to the hard palate of the mouth. With the loop mounted in the mouth and resonant the Voyantic UHF RFID system was used to carry out read range measurements over a range of elevation and rotation angles as shown in Fig. 5, the results of which are given in Table I. For all measurements the mouth was kept closed and the tongue in a neutral position. 


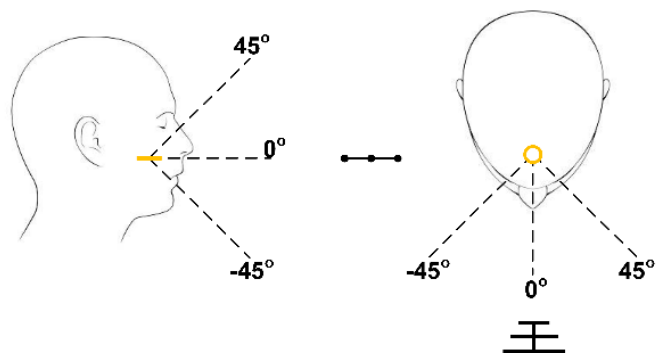

Fig. 5. Elevation and rotation angles in relation to the STL under test

TABLE I.

\begin{tabular}{|l|c|c|c|c|c|c|c|c|c|}
\hline \multicolumn{1}{|c|}{ Ele vation/Rotation Angle (degrees) } \\
\hline \hline \multirow{2}{\text{Read}}{$\begin{array}{l}\text { Range } \\
\text { (meters) }\end{array}$} & $-45,-45$ & $-45,0$ & $-45,45$ & $0,-45$ & 0,0 & 0,45 & $45,-45$ & 45,0 & 45,45 \\
\cline { 2 - 9 } & 0.2 & 0.7 & 0.3 & 0.5 & 0.8 & 0.5 & 0.3 & 0.7 & 0.3 \\
\hline
\end{tabular}

\section{CONCLUSIONS}

This paper has introduced STLs as a potential for use in UHF RFID far-field applications. A prototype targeted at the European RFID band was simulated and measured. This gave promising performance and compares well in terms of read range from previously published work [2], particularly considering, not only the challenges of epidermal mounted antennas but also the RF hostile environment of the oral cavity. In this instance a single loop has been evaluated for RF performance with a conventional asset tag type RFID device but work is ongoing not only to add functionality with some of the sensor type RFID devices but at the same time improve RF performance.

\section{ACKNOWLEDGMENT}

This research was supported by EPSRC project EPM/M025543/21

\section{REFERENCES}

[1] J. S. Belrose, "Performance Analysis of Small Tuned Transmitting Loop Antennas Evaluated by Experiment and Simulation [Antenna Designer's Notebook]," in IEEE Antennas and Propagation Magazine, vol. 49, no. 3, pp. 128-132, June 2007.

[2] O. O. Rakibet, R. J. Horne, S. W. Kelly and J. C. Batchelor, "Passive wireless tags for tongue controlled assistive technology interfaces," in Healthcare Technology Letters, vol. 3, no. 1, pp. 72-76, 32016.

[3] J. C. Batchelor, O. O. Rakibet, C. V. Rumens and S. J. Holder, "Skin-mounted RFID sensing tattoos for assistive technologies," 2014 IEEE MTT$S$ International Microwave Workshop Series on RF and Wireless Technologies for Biomedical and Healthcare Applications (IMWS-Bio2014), London, 2014, pp. 1-3. 